\title{
COMUNICAÇÃO
}

\section{PROCEDÊNCIA DE PACIENTES PORTADORES DE LEISHMANIOSE CUTÂNEA DIFUSA (LCD) NO ESTADO DO MARANHÃO - BRASIL}

\author{
Ana Cristina R. Saldanha, Ana Carla M. e Silva, Cloves Eduardo S. Galvão, \\ Conceição de Maria P. e Silva e Jackson Maurício L. Costa
}

Após os primeiros relatos da leishmaniose cutânea difusa (LCD) na América Latina entre os anos de 1945 e $1950^{3415}$, o Maranhão já na década de 1960, despontava como um dos estados do Brasil, onde ocorria a doença, destacando-se entre os demais pelo número de casos existentes ${ }^{12} 14$.

Embora a leishmaniose tegumentar americana seja pouco estudada no estado, o Ministério da Saúde, através da Fundação Nacional de Saúde (FNS) relatou que entre os anos de 1980 e 1990 foram notificados 16.983 casos, representando $10,2 \%$ da casuística nacional nesse mesmo período, correspondendo a $30,4 \%$ dos casos registrados na região nordeste. Somente no ano de 1990 foram notif cados 3.117 casos de leishmaniose tegumentar americana, sendo o $3^{\circ}$ estado do Brasil com maior frequência (JB Vieira, DIENF, FNS, informação pessoal, 1990).

No que diz respeito à $L C D$, observa-se que desde $o$ aparecimento do primeiro caso da doença em 1960, outros relatos foram feitos, sendo que até a presente data consta nos levantamentos realizados por nossa equipe nos hospitais de São Luís e na Fundação Nacional de Saúde (FNS), regional do Maranhão, um total de 10 (dez) pacientes, representando a maior casuística entre os estados brasileiros, tendo em vista ser uma doença considerada rara pelos autores 6121317 .

Segundo Lainson e Shaw ${ }^{10} 11$, o agente responsável pela $L C D$ em nosso país é a Leishmania (leishmania)_amazonensis e o principal transmissor éo Lutzomya flaviscutellata. No Maranhão apesar de poucos relatos, observa-se a presença de ambos, representando talvez os grandes responsáveis pela disseminação da doença no território maranhense 12 81617

Frente às observacões já descritas, pretende-se neste trabalho mapear a procedência dos casos de LCD no estado, correlacionando com a presenca da Leishmania (leishmania) amazonensis. Para tal a Figura 1 e Tabela 1 mostram-nos os municípios de procedência dos 10 (dez) casos relatados até o

Tabela 1 - Detalhes da procedência dos pacientes portadores de leishmaniose cutânea difusa no Estado do Maranhão, com os respectivos autores responsáveis pelos relatos.

\begin{tabular}{llccl}
\hline Paciente & \multicolumn{1}{c}{$\begin{array}{c}\text { Procedência } \\
\text { (Município) }\end{array}$} & Ano do relato & $\begin{array}{c}\text { Caracterização } \\
\text { do parasita }\end{array}$ & \multicolumn{1}{c}{ Relator do caso } \\
\hline $1^{*}$ & $*$ & 1960 & $\tilde{N} R$ & Porto Marques e cols $^{14}$ \\
$2^{*}$ & Riachão & 1963 & NR & Nery-Guimarães \\
P.C.S. & Rosário & 1977 & Lla** & Departamento de Patologia da UFMA \\
M.E.C.S. & Santa Luzia & 1981 & Lla & Silva e cols ${ }^{17}$ \\
R.O.F. & Coelho Neto & 1986 & Lla & Fonseca e cols \\
R.N.M.G. & Presidente Vargas & 1991 & Lla & Costa e cols \\
A.A.A & Pinheiro & 1991 & Lla & Costa e cols \\
R.N.P.S. & Bacabal & 1991 & Lla & Costa e cols \\
M.C.M.A. & Urbano Santos & 1991 & Lla & Costa e cols \\
J.R.P. & Turiaçu & 1993 & Lla & Silveira \\
\hline
\end{tabular}

* Não consta identificação

** Leishmania (Reishmania) amazonensis

ÑR Caracterização da cepa não realizada

Departamento de Patologia da Universidade Federal do Maranhão, Sao Luís, MA. Endereço para correspondência: Prof. Jackson Maurício L. Costa. Depto. de Patologia/UFMA. Pça. Madre Deus 02, 65025-560 Sao Luís, MA.

Recebido para publicação em 04/02/92. 
Comunicação. Saldanha ACR, Silva ACM, Galvão CES, Silva CMP, Costa JML. Procedência de pacientes portadores de leishmaniose cutânea difusa (LCD) no Estado do Marañhão - Brasil. Revista da Sociedade Brasileira de Medicina Tropical 25:271-273, out-dez, 1992.

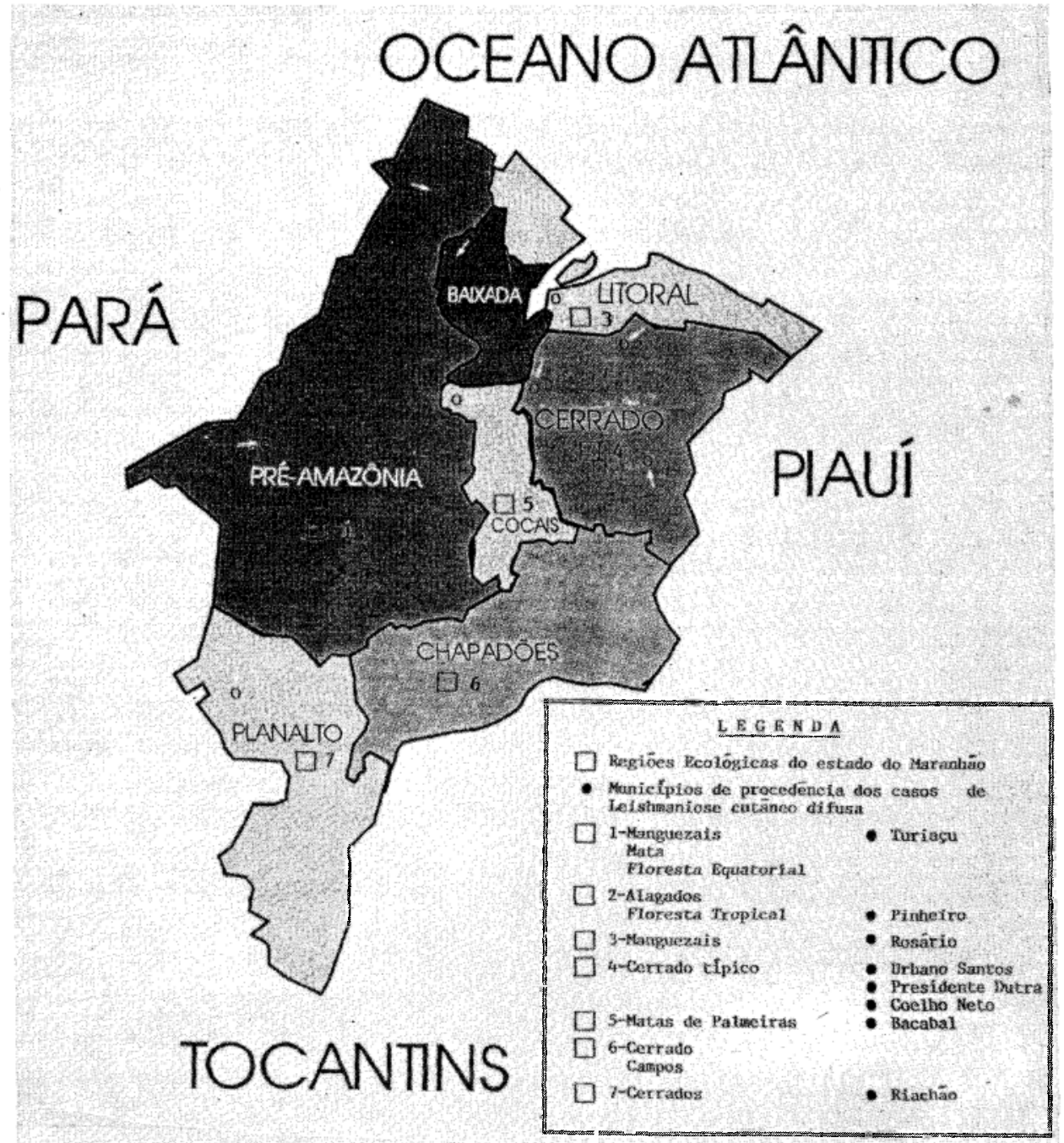

Figura 1 - Mapa do Estado do Maranhäo, mostrando as localidades de onde procedem os pacientes portadores de leishmaniose cutânea difusa (LCD).

presẹte momento. Observa-se que $8(80 \%)$ desses pacientes, são oriundos de regiôes vizinhas, localizadas ao norte e a leste do estado, pertencentes às seguintes regiões ecológicas: Pré-Amazônia $(20 \%)$, baixada $(10 \%)$, litoral $(10 \%)$, cerrado $(30 \%)$ e cocais $(10 \%)$. Em um caso, não obtivemos os dados de procedência (Tabela 1) e apenas um paciente procedia da região ecológica do planalto, localizada mais ao sul e oeste do estado.

Sendo o Maranhão um estado estritamente agrícola, era de se esperar essa diversidade na distribuição dos casos, pois sabemos que a leishmaniose tegumentar é uma doença ligada às áreas de ocupação, e o homem do campo representa o elemento mais importante, sendo o primeiro a entrar em contato com o ciclo silvestre da doença, rompendo o equilíbrio ecológico das florestas primárias, onde existem os mamíferos reservatórios, os insetos vetores e o protozoário, agente etiológico da doença ${ }^{9}{ }^{10}$. Na Figura 1 , observa-se que os pacientes residem em localidades originárias de zonas florestais, isto nos faz pensar que o vetor e o reservatório silvestre da Leishmania (Leishmania) amazonensis em nosso estado possivelmente encontram-se nas áreas florestais ${ }^{12816}$.

\section{REFERÊNCIAS BIBLIOGRÁFICAS}

1. Alvim MC. Distribuicão geográfica dos flebotomíneos do Maranhão. Revista da Sociedade Brasileira de Medicina Tropical 24 (supl II):103, 1991.

2. Alvim MC, Soares OR, Gomes CMC, Costa DR, 
Comunicação. Saldanha ACR, Silva ACM, Galvão CES, Silva CMP, Costa JML. Procedência de pacientes portadores de leishmaniose cutâneo difusa (LCD) no Estado do Maranhão - Brasil. Revista da Sociedade Brasileira de Medicina Tropical 25:271-273, out-dez, 1992.

Martins AMS, Moraes EM, Ahid AP, Ferreira MJS. Composição faunística de flebotomíneos da Illha de São Luís: abundância e ocorrência. In: Resumo do XXVICongresso da Sociedade Brasileira de Medicina Tropical, Natal, p.233-234, 1990.

3. Barrientos PL. Um caso atípico de leishmaniose cutâneomucosa. Memórias do Instituto Oswaldo Cruz 46:415-418, 1948.

4. Convit J, Lapenta P. Sobre um caso de leishmaniose disseminada. Revista de Patologia Clínica (Caracas) 17:153-158, 1946.

5. Costa JML, Saldanha ACR, Pedroso e Silva CM, Serra-Neto A, Galvão CES, Godinho AMR, Silva AC. Estágio atual da leishmaniose cutânea difusa (LCD) no Estado do Maranhão. I. Relato preliminar. Revista da Sociedade Brasileira de Medicina Tropical 24:59-60, 1991.

6. Costa JML, Saldanha ACR, Mello e Silva AC, Serra-Neto A, Galvão CES, Pedroso e Silva CM, Silva AR. Estágio atual da leishmaniose cutânea difusa (LCD) no Estado do Maranhão. II. Aspectos epidemiológicos; clínicos e evolutivos. Revista da Sociedade Brasileira de Medicina Tropical 25:115123, 1992.

7. Fonseca APM, Fonseca WSM. Leishmaniose tegumentar difusa. Relato de um caso. Anais Brasileiros de Dermatologia 61:145-149, 1986.

8. Grimald Jr G, Tesh RB, MacMahon-Pratt D. A review of the geografic distribuition and epydemiology of leishmaniasis in the new world. The American Journal of Tropical Medicine and Hygiene 41:687-725, 1989.

9. Instituto Brasileiro de Geografia eEstatística. Sinopse Preliminar do Censo Demográfico. IX Recenceamento Geral do Brasil - Fundação Instituto Brasileiro de Geografia e Estatística, Maranhão, 1980.

10. Lainson R, Shaw JJ. Las leishmanias y la leishmaniasis del nuevo mundo, con particular referência al Brasil. Boletin de la Oficina Panamericana 76:93-119, 1974.

11. Lainson R, Shaw JJ. Evolution, classification and geographical distribuition In: Peters $\mathrm{W}$, KillickKendrick K (eds). The Leishmaniasis in Biology and Medicine. Academic Press London. p.1-120, 1987.

12. Nery Guimarães F. Estado atual dos conhecimentos da "forma lepromatoide" da Leishmaniose tegumentar (LT). O Hospital 67:71- 92, 1965.

13. Pessoa SB, Barreto MP. Leishmaniose Tegumentar Americana. Imprensa Nacional, Rio de Janeiro, 1948.

14. Porto Marques A, Portugal H. Leishmaniose tegumentar difusa. O Hospital 57:11-23, 1960.

15. Silva F. Forma raríssima de leishmaniose tegumentar. Leishmaniose dérmica não ulcerada em nódulos e extensas placas infiltradas e hiperpigmentadas. Separata do volume da Reunião Anual de Dermatosifilógrafos Brasileiros, Rio de Janeiro, 1946.

16. Silva AR, Martins G, Melo JEM, Araújo JP, Mendes JR, Mendes MG. Surto epidêmico de leishmaniose tegumentar americana ocorrido na Colonização Agrícola de Buriticupu (Estado do Maranhão) Brasil. Revista do Instituto de Medicina Tropical de São Paulo 21:43-50, 1979.

17. Silva AR, Mendes JR, Rodrigues MLM, Carvalho ZS, Reis FMP, Melo JEM, Morais JCO. Leishmaniose cutânea difusa (LCD). Registro de um caso em Bruriticupu (Estado do Maranhão). Revista do Instituto de Medicina Tropical de São Paulo 23:1-4, 1981.

18. Silveira FT. Resultados preliminares do tratamento de dois casos de leishmaniose cutâneo difusa, diagnosticados em diferentes fases evolutivas da doença. In: Resumos do XXIX Congresso da Sociedade Brasileira de Medicina Tropical, Fortaleza p.106, 1993. 